\title{
Laboreal
}

Volume $6 \mathrm{~N}^{\circ} 1$ | 2010

Varia

\section{El ergónomo frente a la crisis global}

o ergónomo face à crise global

L'ergonome face à la crise globale

The ergonomist faced up to the global crisis

\section{François Daniellou}

\section{OpenEdition}

\section{Journals}

Edición electrónica

URL: http://journals.openedition.org/laboreal/9160

DOI: $10.4000 /$ laboreal.9160

ISSN: 1646-5237

\section{Editor}

Universidade do Porto

\section{Referencia electrónica}

François Daniellou, «El ergónomo frente a la crisis global », Laboreal [En línea], Volume 6 NN$^{0} 1$ | 2010 Publicado el 01 julio 2010, consultado el 23 septiembre 2020. URL : http://journals.openedition.org/ laboreal/9160; DOI : https://doi.org/10.4000/laboreal.9160

Este documento fue generado automáticamente el 23 septiembre 2020

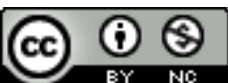

Laboreal está licenciado com uma Licença Creative Commons - Atribuição-NãoComercial 4.0 Internacional. 


\title{
El ergónomo frente a la crisis global
}

\author{
o ergónomo face à crise global \\ L'ergonome face à la crise globale \\ The ergonomist faced up to the global crisis
}

\section{François Daniellou}

\section{NOTA DEL EDITOR}

\section{François Daniellou}

Doctor en Ergonomía (CNAM, 1985). Ingeniero Mecánico ECP (Ecole Centrale de Paris, 1977);

Fundador (1993) y Director del Laboratoire d'Ergonomie des Systèmes Complexes (ISPED);

Miembro del Comité de la Precaución y la Prevención del Ministère de l'Ecologie et du Développement Durable de Francia;

Profesor del Institut Polytechnique de Bordeaux;

ADEA - Asociación de Ergonomía Argentina (Asociación sin fines de lucro);

Miembro federado de la IEA - International Ergonomics Association;

Miembro fundador de ULAERGO Unión Latinoamericana de Ergonomía.

Programa PREFALC

Se trata de un Dispositivo Regional de Cooperación Universitaria en Red Francia América Latina - el Caribe, impulsado por el Ministerio de Asuntos Exteriores y Europeos (MAEE), el Ministerio de la Educación Nacional, la Enseñanza Superior y la Investigación (MEN-ESR) y la "Fondation Maison des Sciences de l'Homme". Su objetivo es "mejorar la movilidad exclusivamente del personal docente" (para) "contribuir al desarrollo de las relaciones internacionales con los países de América Latina en el ámbito de los intercambios universitarios y científicos, así como de participar en el surgimiento de un espacio de enseñanza superior Unión Europea América Latina - El Caribe". 
Este objetivo fue reafirmado en la "Cumbre de jefes de Estado de la zona UEALC" en Viena (mayo 2006).

\section{NOTA DEL AUTOR}

Texto del Profesor François Daniellou en su conferencia como único orador en el Salón Auditorio de la Universidad Tecnológica Nacional - Regional Buenos Aires, el miércoles, 27 de mayo de 2009. El evento fue organizado por la Asociación de Ergonomía Argentina (ADEA), en el marco del Programa PREFALC. propusieron plantea la responsabilidad de los ergónomos frente a la crisis internacional. Aunque podemos hacer poco, lo que sí podemos es intentar con afán que el tema del trabajo humano, de las condiciones de trabajo y de sus consecuencias sobre la salud no desaparezcan del análisis de lo que está sucediendo.

3 Mis percepciones de la crisis en Francia están muy influenciadas por un trabajo que realizamos junto con Philippe Davezies y Karine Chassaing, para la empresa consultora Emergences y el sindicato CGT de los trabajadores de la Renault. Ese trabajo empezó con Jacques Duraffourg a principios de 2008. Se trataba de ayudar a los equipos sindicales a enfrentar el tema de los riesgos psicosociales, después de una serie de suicidios sucedidos en la empresa. A partir de octubre de ese año tuvimos que enfrentarnos a la crisis y a la clausura provisoria de muchas plantas, al desempleo parcial. También tuvimos la oportunidad de descubrir algunas de las medidas tomadas por la empresa, que usó la crisis como un laboratorio social al revés, como una forma de comprobar hasta dónde se puede ir en materia de flexibilidad. saber si van a trabajar la semana siguiente. En febrero trabajaron 6 días. Si trabajan, van a ser asignados a un puesto de trabajo que no conocen, en una línea de montaje desconocida, en un equipo que no es el suyo. Ensamblarán partes de automóviles, ya sea con un ritmo aumentado o disminuido, pero teniendo que ocupar tres puestos de trabajo en vez de uno. Si la empresa no los necesita la semana siguiente, tal vez los llamarán para que trabajen un sábado, o tal vez se incremente el número de días negativos en su contador individual.

5 Los días negativos son los días que tendrán que devolver a la empresa, días que tendrán que reintegrar si son despedidos. Muchos trabajadores ya tienen 90 días negativos.El desempleo parcial no es un tiempo de descanso, es un tiempo muerto, un tiempo de ruptura de las solidaridades y de los recursos colectivos, un tiempo de recogimiento en sí mismo, en el cual, para enfrentar miedo del día siguiente, la cognición humana puede detenerse y concentrar el odio en el colega, en el compañero en la línea de montaje, que ha tenido la suerte de trabajar dos días más durante el mes pasado.

6 Como lo recordaba Michel Serres [1], "crisis" tiene el mismo origen que "cribar". De lo que se trata con la raíz "cri", "cribar", "la crítica", "los criterios" es de escoger, de dividir lo que vale y lo que no vale, las lentejas y las piedras, los conceptos operativos y los lugares comunes, lo que uno conserva y lo que se descarta. Es una familia lingüística que invita a discernir y a zanjar. La crisis médica es una bifurcación: o el organismo se 
muere o inventa una forma de sanar que nunca será una mera vuelta a lo anterior. La crisis económica prohíbe la conservación de lo mismo: no se puede volver a la situación anterior. Pero no estamos aquí para especular sobre el futuro, sino para compartir sobre nuestras responsabilidades, nuestras elecciones profesionales.

7 Les propongo tres partes en esta presentación. Para empezar, miremos al pasado, a la evolución del entorno económico y de las organizaciones laborales. En un segundo momento, hablaremos de la situación presente y en particular del sufrimiento psíquico de los trabajadores. $Y$ terminaremos por algunos temas que nos conciernen directamente, en tanto ergónomos.

¿Cómo llegamos hasta aquí? Los ergónomos necesitamos dejar nuestro microscopio y ensanchar nuestra mirada con la ayuda de los economistas. Lo que voy a presentar es una historia más europea en la cual uso particularmente un texto de Philippe Davezies. Les dejo evaluar lo que también tenga de pertinente para América Latina.

El 22 de julio de 1944, 6 semanas después del desembarco en Normandía, los delegados de 44 países - Keynes estaba al frente de la delegación británica - firmaban en Bretton Woods el acuerdo fundador del orden económico de la posguerra. El liberalismo sin límites del inicio del Siglo XX había llevado a la crisis de 1929 y a la Segunda Guerra Mundial. De lo que se habla por entonces es de regulación económica: se establecen tipos fijos de cambio y control de los mismos, los bancos centrales tienen poder, se funda el FMI.

10 En el medio industrial, los treinta años que siguieron fueron una época marcada por un compromiso Fordista entre el capital y el trabajo, entre los patrones y los sindicatos, con el arbitraje del Estado, financiado por el crecimiento monetario. Pero en el periodo 1965-1970 el modelo keynesiano se agrieta. Estados Unidos, por las "necesidades" de la guerra en Vietnam y de la conquista del espacio, saca el dólar de las reglas de paridad que habían sido establecidas con el oro. Esto desemboca en una fuerte inflación en los países desarrollados, y en 1973 trae como consecuencia el fin del sistema de tipos fijos de cambio.

11 De ahora en adelante es el mercado quien lo definirá. La debilitación del desarrollo y los choques petroleros suceden al mismo tiempo. Las crisis sociales se multiplican.

12 A finales de los setenta la ofensiva liberal se desencadena. Había sido preparada ya desde finales de los cincuenta, particularmente en los think tanks ultraliberales, alrededor de Milton Friedman y de Friedrich Hayek, en la Universidad de Chicago.

13 Con fundamentos distintos se oponían a la intervención estatal en la economía y a toda regulación, y preconizaban el dejar hacer. Sus teorías fueron probadas en gran escala en Chile por los Chicago Boys, sus estudiantes chilenos que aconsejaron a Augusto Pinochet a partir de 1973.

14 Las mismas teorías influenciaron a Ronald Reagan y a Margaret Thatcher a principios de los ochenta. Todo obstáculo a la circulación del capital se elimina, las inversiones se globalizan y se vuelven principalmente especulativas. El 5 de agosto de 1981, Reagan despide a 11000 controladores aéreos huelguistas y los reemplaza por militares hasta que sus sucesores hayan sido formados. En marzo de 1985, Margaret Thatcher doblega a los mineros británicos después de casi un año de huelga. El liberalismo más desenfrenado llega a ser la regla en la mayoría de los países desarrollados y en países emergentes, imponiéndoselo a los países más pobres.

Durante ese período, ¿qué sucede con relación al trabajo? 
16 El Fordismo de los años treinta de la posguerra organizaba una actividad previsible, planificada desde abajo. Se trataba de un mercado del primer equipo: el primer auto, la primera lavadora, el primer televisor. La oferta generaba la demanda a partir de una cierta prosperidad y el consumidor compraba lo que la industria producía. Las variantes eran limitadas. Los departamentos de métodos definían el one best way productivo, los asalariados y sus representantes negociaban contratos considerables, por lo menos para los hombres asalariados calificados. Las jefaturas contribuían para enmarcar los procesos de trabajo.

17 Pero, con el debilitamiento del desarrollo en los años 70-80, ya no se pueden vender productos que fueran para tomar o para dejar. Las variantes se multiplican, la producción vuelve a regirse por la demanda, la flexibilidad y el poder de respuesta devienen los desafíos mayores. No se pueden alcanzar por un mero perfeccionamiento de lo que ya existe. La iniciativa y la movilización subjetiva de los trabajadores son imprescindibles. El metodólogo cede el lugar al gerente: es la movilización de las energías de los trabajadores y no la prescripción detallada del trabajo la que prepondera en el discurso gerencial.

Desde nuestro punto de vista de ergónomos, estos cambios podrían ser considerados como parcialmente positivos: se requiere de forma explícita la inteligencia de los asalariados, se reconoce el rol de la subjetividad individual. Pero ese optimismo no dura: el riesgo de que la mejora de los resultados suponga una pérdida del poder de control social aparece de forma clara para los mandos. Las empresas, especialmente las francesas, no desean perder el control, aún siendo para ganar dinero. En los 90, los procedimientos afluyen, sobre todo en los ámbitos de la calidad, del control de gestión, de la trazabilidad. Invaden sectores hasta entonces poco Tayloristas, como los hospitales y los centros de atención al cliente. El trabajo se intensifica debido a la sumatoria de requerimientos industriales y comerciales. Cada unidad de producción vuelve a ser cliente de la anterior y proveedor de la siguiente. Los intercambios entre unidades y la valoración global del sistema se basan en indicadores cada vez más alejados de la realidad del trabajo.

19 La gestión se convierte en un mundo hechizado, un mundo de cristal, desconectado del funcionamiento de los talleres, poblado de profesionales de las finanzas sin experiencia industrial, que optimizan para los accionistas los beneficios a corto plazo y que entienden la operación de la empresa por medio de números... alimentados por sistemas de información programados para subir solamente información sobre el alcance de los objetivos. Lo que se necesitó para alcanzarlos, el costo humano de la producción, nunca llega a la sede social. Cuando el director venga a la planta se ordenará el taller y se pintarán como nuevos los corredores por los que transitará.

20 Este mundo de cristal, completamente desconectado de la economía real, se rompió hace algunos meses arrastrando al planeta a una vorágine sin precedentes. Pero nosotros, que estamos en los talleres de producción, en los hospitales, tenemos todas las razones para pensar que las mismas causas pueden acarrear las mismas consecuencias en el mundo industrial y en el de los servicios. Ya no hay casi ninguna relación entre lo que sucede en las situaciones de trabajo y los discursos generales que escuchamos sobre la buena marcha de la empresa o de la planta. Ya no hay casi ninguna relación entre las tablas informatizadas que son llenadas por los gerentes y la realidad del funcionamiento de los servicios. La mayoría de los números son engaños por su naturaleza conceptual, y otros son distorsionados para cumplir con las expectativas. 
Cada semana vemos situaciones de origen organizacional en los talleres, que muestran la enorme dificultad que tienen los trabajadores para alcanzar el nivel de producción requerido, y que son arregladas por los asalariados y por los mandos medios, y encubiertas por un discurso suave sobre las orientaciones de la empresa que supuestamente está bajo control, como lo garantizan los consultores y auditores que pasan frecuentemente, con los ojos cerrados y la boca abierta.

21 Ahora bien, y en eso nosotros estamos directamente implicados, el nudo del asunto es la calidad y, de manera más precisa, la calidad del trabajo bien hecho. Pues de un lado, el del mundo de cristal, existe la "calidad para el mercado, en el tiempo del mercado". La excelencia es hacer "justo lo necesario" para aguantar mejor que los competidores. Ustedes llaman al servicio de atención al cliente de su operador de Internet y, si no arreglan su problema dentro de tres minutos, tal vez van a proponerles ponerlos en contacto con el departamento de rescisión del servicio. Es más fácil perderlos como clientes que tratar de solucionar su problema y pasa lo mismo, de todas formas, con la competencia. La excelencia vista por los gerentes es no hacer más de lo que se necesita para ser competitivo, y el nivel de referencia se define por los procedimientos. La excelencia vista por los gerentes es una optimización de variables que no suponen ninguna diferencia por su naturaleza, ni ningún conflicto entre las dimensiones que agregan acerca del equivalente monetario general.

Del otro lado de la calidad se encuentran mujeres y hombres que intentan hacer bien su trabajo. Están en el lugar adecuado para conocer la variabilidad de la producción y cuidar de ella. Intentan dar una respuesta distinta a configuraciones que cambian, a fin de que el producto o el servicio salgan de forma correcta. Son incansables cuando hablan de las diferencias entre piezas, entre clientes, entre llamadas, entre pacientes. Cuidan, eso significa también que están confrontados cotidianamente con debates sobre normas, con contradicciones minúsculas y mayores entre dimensiones de eficacia y dimensiones de ética. Por supuesto, "cuidar" una llamada, "cuidar" un paciente, "cuidar" un automóvil por arreglar, eso requiere un poco de tiempo. Si "cuidan" demasiado podría ser que su entrevista anual de valoración resulte tensa: ¿por qué son tan poco productivos? Muy a menudo la Dirección, que ahora se elige por su capacidad para no conocer el trabajo, no entiende las explicaciones detalladas que el trabajador intenta aportar, y lo llama al orden: los ratios son malos, tienen que ser buenos y basta. El trabajador se encuentra digitalizado según la expresión de Jean-Louis Malys. Esta situación de valoración meramente cuan- titativa también alcanza a los investigadores y a los ergónomos en algunas condiciones de empleo. Todos estamos más o menos digitalizados.

Me dirán: ¿y Kaizen? ¿Los talleres Kaizen son una manera de escuchar a los trabajadores, de sacar provecho de sus conocimientos sobre la variabilidad? En el Toyota Production System original sí, indudablemente. Toyota ha entendido de forma profunda la necesidad de empalmar dos tipos de conocimientos, los conocimientos generales de los expertos, que permiten anticipar por reglas y dispositivos lo que va a pasar, y los conocimientos específicos de los asalariados, quienes cuidan de situaciones particulares, estos conocimientos que permiten hacer frente a la variabilidad, a lo imprevisto. El Toyota Production System presentó un equilibrio largamente madurado y hondamente arraigado en la cultura japonesa, en el cual los trabajadores eran invitados a depositar estos conocimientos, considerados como inmensamente 
respetables en el acervo general de la empresa, a cambio de muchas garantías y contraprestaciones.

Las versiones del TPS que nos presentan en Francia, con el telón de fondo de las "high greed organisations", las organizaciones codiciosas que quieren todo de golpe, poco tienen que ver con el original: un taller Kaizen de tres días, o incluso de tres horas, es una forma de obligar a los trabajadores a utilizar sus conocimientos para alcanzar objetivos decididos con antelación, y que siempre suponen disminuir el tiempo y el espacio. Si, como ocurre a menudo, la situación resulta peor después, no tendrán ningún derecho a criticar: se siguieron sus ideas.

Entre los países ricos de Europa occidental, Francia es el país en el cual los trabajadores se sienten menos escuchados y menos respetados. También es donde la diferencia es mayor entre los ingresos más altos y los más bajos. El país de los Derechos Humanos, es un país de desigualdades sociales crecientes y de diálogo social atascado.

Luego, por todas partes, surgen los Trastornos MúsculoEsqueléticos o se habla de riesgos psicosociales. Los TMEs, como saben, afectan a los asalariados que son bloqueados, que no tienen los márgenes necesarios de maniobras individuales y colectivos para construir comportamientos profesionales que encarnasen sus competencias y los protegiesen, en organizaciones en las cuales, por lo general, su jefe y el jefe de su jefe también son bloqueados.

27 ¿Y los riesgos psicosociales? Han notado que ahora se habla mucho de riesgos psicosociales como si se tratara de una nube tóxica, cernida sobre la empresa, o sobre uno de sus departamentos, y que afectaría a algunos de los asalariados y en primer lugar, por supuesto, a aquellos cuyas características individuales los fragilizan.

Según este modelo, el clima está un poco podrido y los más sensibles van a tener problemas: se hacen análisis del clima laboral (se mide el entorno psicosocial). Se hacen dosificaciones en los empleados (para evaluar el riesgo psicosocial que corren). Se puede establecer un sistema de alerta mutua (advertir cuando un colega no está bien o si se lo considera frágil). Se puede intentar desintoxicar a los ya afectados, por ejemplo, brindándoles un apoyo psicológico financiado por la propia empresa como cuando se coloca en la cámara hiperbárica a las personas afectadas por el monóxido de carbón. Por todos los medios se intenta evitar que la nube genere demasiadas victimas, sobre todo las que tienen una fragilidad intrínseca que podría llevarlas hasta el intento de suicidio, que acarrea consecuencias letales para la organización y la imagen de marca.

Este modelo está a punto de llegar a ser consensuado en muchos sitios: frente a los dramas ya acaecidos, los sindicatos están aliviados cuando los directivos deciden hacer algo con la garantía de especialistas externos. No escasean los panfletos sindicales con desarrollos eruditos sobre la medida del estrés, que repiten con muy buena fe los mismos argumentos de los que aparecen en las presentaciones de la Dirección.

Ahora bien, si se puede entender que exista un cierto consenso conceptual cuando se trata de enfrentar el riesgo radioactivo o cualquier riesgo toxico, el consenso es mucho más problemático cuando se refiere a los riesgos psicosociales. Para tomar una definición de Philippe Davezies, los riesgos psicosociales son los que resultan de la confrontación entre el funcionamiento social, de la organización; y el funcionamiento psíquico, de la persona.

31 Hoy, del lado de la persona, ya lo hemos dicho, el individuo es tomado como un eslabón intercambiable, la prescripción de la calidad para el mercado, las órdenes 
contradictorias, los dobles vínculos entre el cumplimiento de procedimientos y la incitación a la chapucería, el desprecio a la inteligencia y a los conocimientos locales, los niveles de gestión basados en el dinero como equivalente general, y las valoraciones personales sin relación con la dificultad del trabajo, que destruyen los colectivos, ponen a competir a los trabajadores, y los aíslan.

Del lado de las personas, el funcionamiento psíquico que supone no ser tratado como un objeto sino como una persona, disponer de una cierta autonomía, poder utilizar las competencias que uno ha desarrollado a partir de la confrontación cotidiana con el cambio, aprender cosas nuevas, poder influir en su entorno, poder deliberar acerca de las contradicciones cotidianas entre eficacia y ética, tejer vínculos de ayuda mutua y de solidaridad con sus allegados, y debatir con los colegas sobre las reglas del oficio.

Cuando las exigencias de estos dos funcionamientos son contradictorias, los empleados sufren, los conflictos se multiplican. Pero la imposibilidad de entenderlos como consecuencia del trabajo, desemboca en atribuirlos a la personalidad de los demás: el acosador es el jefe o son los colegas, no la organización laboral. Cada uno se encuentra aislado en su enfrentamiento al absurdo, pues la vergüenza del trabajo mal hecho no es un sentimiento que se comparte.

Pero, y en eso tenemos nuestro rol propio, estos conflictos entre el funcionamiento de la organización y el de la persona se evidencian de forma distinta en cada situación de trabajo: hacer bien su trabajo de asistente, hacer bien su trabajo en el departamento de compra, hacer bien su trabajo de dibujante, son cosas diferentes. No poder hacer bien su trabajo no se juega de la misma forma para la asistente, el comprador o el dibujante. Tal vez los metrólogos de la nube psicosocial han detectado que estos tres empleados están mal. Pero existe la duda que se mejore algo si no se mira con detalle, si no se habla de lo que está en juego en la oficina, en el departamento de ventas, en el de diseño: ¿Cuáles son los conflictos de lógicas no debatidos que llevan a la persona a sentir que no puede hacer lo que habría de hacer para que esté bien hecho, que no puede desarrollarse, que se aísle de sus colegas? ¿Qué está en juego en sus relaciones cotidianas con el objeto de su trabajo y con los discursos sobre dicho objeto enunciados por la organización laboral? ¿Cómo los mandos medios del departamento se encuentran atrapados entre las órdenes descendientes y una realidad del terreno que no pueden reportar y que, por lo tanto, le puede resultar más fácil de no percibir desde un punto de vista psíquico? ¿Cómo el patrón de una pequeña empresa se encuentra asfixiado por los procedimientos de sus clientes, para quienes el tema de lo realizable simplemente no se plantea?

Esa situación dramática de los asalariados franceses, incluso los directivos, interpela a los ergónomos. Los temas del trabajo y de la salud laboral no son un lujo que se podría dejar de lado en tiempo de crisis. De la crisis no todas las empresas saldrán ilesas, muchos trabajadores ya han perdido o van a probablemente perder su empleo. Algunas empresas piensan que el camino de la supervivencia pasa por un refuerzo de las órdenes descendientes, del control, y en algunas de ellas, del desprecio. Podríamos estar tentados de esperar a que la crisis pase. Pero tenemos que afirmarlo, basándonos en nuestro patrimonio profesional: la crisis industrial es una crisis de pérdida del contacto con la realidad del trabajo y con los objetos de trabajo, una crisis de los enfoques totalmente top down, de un funcionamiento en el cuál sólo valen las informaciones descendientes, una crisis del mundo de cristal alimentado por visiones y niveles desconectados de la realidad, una crisis de los modos de gobierno basados en la 
omnipotencia del cálculo anticipado, en el desprecio a la inteligencia y a la capacidad creativa de los trabajadores.

La única salida, vista desde mi humilde ventana de ergónomo, es un nuevo arranque de la democracia industrial, la emergencia de formas de dirección de las empresas que den lugar al respeto y a los debates entre diferentes lógicas. El desafío es plantear nuevas formas de confrontaciones entre los conocimientos descendientes, los conocimientos de los expertos que permiten anticipar y preparar los medios de trabajo; y los conocimientos relacionados con la experiencia, los conocimientos de los cuerpos y de los colectivos que permiten enfrentar a lo que no es dado con antelación por la organización laboral, de enfrentar lo imprevisto, de contestar a la realidad por compromisos elaborados. Estas dos fuentes de conocimiento son imprescindibles y deben articularse lo más cerca posible de la realidad del trabajo. El desafío es que los conflictos de las lógicas, que son normales y ineludibles pues la empresa es el trabajo en el que conviven lógicas que no tienen ninguna razón de convivir, sean expresados y debatidos, y que las salidas sean negociadas. El desafío es que los directivos vuelvan a encontrar la misión, los medios y el afán de hacer vivir, a cada nivel y en ambos sentidos, la articulación entre las orientaciones de la empresa y la realidad del terreno. Mejorar los resultados no significa aumentar el ritmo de trabajo, ni encontrar una zona del mundo en la cuál los trabajadores cuestan menos, sino que mejorar los resultados significa darse cuenta del gigantesco desorden que representan muchas organizaciones, tomar consciencia de la futilidad de los indicadores de gestión, del tiempo laboral más dedicado a reportar "sin novedades, jefe" que a enfrentar la realidad que existe. Mejorar los resultados significa tomar consciencia del carácter artificial de los mensajes empresariales dominadores y de la necesidad de hacerlos más humildes. Mejorar los resultados es acercarse a la realidad cotidiana, con los ojos y los oídos abiertos.

Luchar contra los riesgos psicosociales no significa brindar psicoterapias a los asalariados cuya subjetividad se ha estrellado, sino reconocer que cada uno de ellos tiene un rol específico por desempeñar en los pliegues de la empresa, con la condición de que se le permita trabajar con sus colegas y sus jefes en la elaboración de las condiciones de un trabajo bien hecho.

39 Frente a los riesgos psicosociales, los ergónomos estamos divi didos. Algunos piensan que no nos compete a nosotros, sino a los psicólogos. Algunos utilizan, con más o menos suerte, los conceptos y métodos de la psicodinámica o de la clínica del trabajo. Personalmente, la confrontación con esta forma de sufrimiento me incita a aproximarme aún más a la actividad de trabajo que suelo hacer cuando participo en proyectos industriales.

40 La mirada modesta, curiosa y benévola que nos enseño Jacques Duraffourg, esta forma de mirar y de escuchar desde el "punto de vista del trabajo", es un analizador muy potente de la situación económica y sanitaria del mundo laboral. Probablemente es el único que permite volver a traer la realidad, en el mundo hechizado, el mundo de cristal del cual acabamos de hablar. Esta mirada, esta escucha, permiten detectar las contradicciones locales, los conflictos de lógicas, los debates necesarios, en un entorno en el cual se preconiza el consenso a priori; y que provoca oposiciones globales.

41 Las situaciones favorables son aquellas en las que existen simultáneamente valores compartidos acerca de la calidad del trabajo y debates sobre lo que su realización presupone. 
Es nuestra responsabilidad compartir esta forma de mirar, particularmente con los representantes sindicales y con los directivos. Es un camino largo. Los representantes sindicales, como los directivos, muy a menudo piensan conocer la realidad del trabajo de los empleados que están encargados de representar o de dirigir. Ambos, se encuentran movilizados en el frente económico; y la actividad que tiene lugar en los puestos de trabajo no es su primera preocupación.

Sin embargo, cuando se puede iniciar este camino, cuando se puede incitar a los mandos y sindicalistas a ir a ver el trabajo de cerca, puede suceder un encadenamiento que merece mencionarse:

- Los trabajadores cuyo trabajo ha sido analizado descubren, en la descripción que se hace del mismo, los conflictos de lógicas y las regulaciones contradictorias que pesan sobre su trabajo y que les impiden estar orgullosos de su resultado. Sospechan que el origen de sus dificultades no es una fragilidad personal, ni la personalidad de su jefe, sino debates de lógicas que no tienen sentido.

- Si empiezan a hablar de esto con sus colegas, se puede crear un potencial de comprensión y de solidaridad colectivas. Talvez el grupo sea entonces capaz de formular reivindicaciones concretas, que tienen una oportunidad de tener éxito.

- Si tal es el caso, si vuelven a retomar el dominio de algunas dimensiones de su situación de trabajo, su poder de actuar que hasta entonces estaba deteriorado, podrá desplegarse en temas de mayor alcance.

Este camino, lo hemos vivido con sindicalistas, tal como lo contamos en "Le travail intenable" [2]. Pero también lo hemos vivido con jefes de servicio, responsables de relaciones humanas, o directores de planta.

Volver a introducir el tema del trabajo en los debates acerca de los resultados de la empresa significa, por supuesto, alertar sobre los costos humanos de los resultados, y sobre las consecuencias sobre la salud de los trabajadores. Pero también significa volver a tejer el vínculo entre el nivel micro y el macro, enseñar cómo el valor agregado se crea en el detalle de la gestión local de la variabilidad, en la forma de cuidar los sucesos microscópicos, cómo intentos de hacer bien resultan esterilizados por la algarabía empresarial y la sordera organizacional.

Tenemos que hacer la prueba, en casos limitados, de lo que se puede ganar tomando en serio los conocimientos de las personas y de los colectivos, y propiciando sus interacciones con los conocimientos generales de los expertos. Estoy convencido de que nuestra capacidad de intervenir en proyectos técnicos y organizacionales es una ventaja, si no la usamos solamente como una pericia técnica sino, también, como una oportunidad de volver a introducir un poco de democracia social en los procesos de diseño.

4 Después de la crisis, los demógrafos lo anuncian, vendrá en Europa el tiempo del déficit de mano de obra calificada. Las empresas tendrán que merecer a sus trabajadores, una situación que siempre ha propiciado el desarrollo social en los países nórdicos. Eso conlleva responsabilidades para los empleadores, los sindicatos, el Estado, y también para nosotros.

Como lo he dicho al inicio, acabo de presentarles un punto de vista francés.

Ahora espero compartir con vosotros acerca de la realidad latinoamericana, y de sus posiciones sobre el tema. Gracias por su atención. 


\section{NOTAS}

1. Michel Serres et Michel Polacco, La crise, in Le sens de l'info, France Inter, 8 Février 2009

2. Théry, L. (2010). Le travail intenable (2ème edition). La Découverte: relato de una experiencia de 2 años con 23 equipos sindicales de CFDT que formamos para el análisis de situaciones de trabajo, para una mejor comprensión de lo que significaba la intensificación del trabajo en su empresa.

\section{AUTOR}

\section{FRANÇOIS DANIELLOU}

Département d'Ergonomie - École National Supérieure de Cognitique Institut Polytechnique de Bordeaux, Case 55, 146, rue Léo-Saignat, 33076 BORDEAUX Cedex

francois.daniellou@ensc.fr 